\title{
Gesellschaftliche Wertschöpfung klassischer Massenmedien: Die Perspektiven von Medienorganisationen und Publikum in sechs Europäischen Ländern
}

\author{
Krebs, Isabelle ; Russi, Loris ; Siegert, Gabriele
}

Posted at the Zurich Open Repository and Archive, University of Zurich ZORA URL: https://doi.org/10.5167/uzh-65224

Book Section

Published Version

Originally published at:

Krebs, Isabelle; Russi, Loris; Siegert, Gabriele (2012). Gesellschaftliche Wertschöpfung klassischer Massenmedien: Die Perspektiven von Medienorganisationen und Publikum in sechs Europäischen Ländern. In: Kolo, Castulus; Döbler, Thomas; Rademacher, Lars. Wertschöpfung durch Medien im Wandel. Baden-Baden: Nomos, 361-378. 


\title{
Gesellschaftliche Wertschöpfung klassischer Massenmedien
}

\section{Die Perspektiven von Medienorganisationen und Publikum in sechs Europäischen Ländern}

\author{
Isabelle Krebs / Loris Russi / Gabriele Siegert
}

\section{$1 \quad$ Einleitung}

Die Feststellung, Medien seien gleichzeitig Kultur- und Wirtschaftsgüter, basiert auf zwei unterschiedlichen funktionalen Bezugsrahmen moderner Gesellschaften, der marktwirtschaftlichen Organisation des Mediensystems einerseits und den Massenmedien als zentralen Selbstbeobachtungsinstanzen der Gesellschaft andererseits. Die daraus abgeleiteten ökonomischen und publizistischen Funktionen korrespondieren mit den Ansprüchen an die Wertschöpfung eines Mediensystems. Der Wertschöpfungsbegriff schließt unmittelbar hier an. Die Debatte zum Begriff Public Value und dessen Institutionalisierung in politischen Prozessen wie den Dreistufentests können als Beispiele für die Relevanz gesellschaftlicher Wertschöpfung angeführt werden. In eine ähnliche Richtung gehen auch die Forderungen der Verleger nach einem Leistungsschutzrecht mit der Begründung, dass die Presse im Besonderen, aber auch die anderen traditionellen Massenmedien, Träger und Verfechter gesellschaftlicher Wertschöpfung seien.

Vor dem Hintergrund technologischer und struktureller Veränderungen der Medienbranche und des Nutzungsverhaltens ist daher die Frage zu klären, ob diese Rollenzuschreibungen in der Branche entsprechend vertreten, umgesetzt und wahrgenommen werden. Konkret gilt es gerade auch unter dem Eindruck der Wirtschaftskrise zu ermitteln, inwieweit ökonomische Ziele und Marktorientierung mit dem gesellschaftlichen Auftrag vereinbart werden. Der vorliegende Beitrag widmet sich diesem „erweiterten“ über ein rein ökonomisches Verständnis hinausgehenden Wertschöpfungsbegriff und beschäftigt sich einerseits mit der Frage, welche Ziele Medienorganisationen verfolgen und inwieweit hierbei Funktionen im Sinne gesellschaftlicher Wertschöpfung Berücksichtigung finden und andererseits, wie die Leistung und Funktionserfüllung durch die Bevölkerung beurteilt wird. Um die Wertschöpfung eines Mediensystems abbilden zu können, wird also nicht nur die Produzenten-, sondern auch die Publikumssicht berücksichtigt. 
Zu diesem Zweck wurden in sechs europäischen Ländern sowohl Medienverantwortliche zu den Funktionen ihrer Medien und zu ihren konkreten Zielen sowie das Publikum zur Leistung und Funktionserfüllung von Medienorganisationen befragt.

\section{Theoretische Grundlagen}

Funktionen und damit verbundene gesellschaftliche Wertschöpfung sind Aufgaben, die Massenmedien erfüllen sollen (normativ, vgl. z.B. Burkart 2002: 390ff.). Sie beschreiben aber ebenso Leistungen, die real erbracht werden (vgl. Bonfadelli 2010: 134; Ronneberger 1974: 198). Die gesellschaftliche Wertschöpfung beschreibt daher zwei Seiten einer Medaille: Der Soll-Wert im Sinne von Ansprüchen an ein Mediensystem und der Ist-Wert als tatsächlich erbrachte Leistungen. Aus demokratietheoretischer Sicht sollten Anspruch und Leistung möglichst übereinstimmen (vgl. McQuail 1992).

Die gesellschaftlichen Ansprüche an Massenmedien sind in sogenannten Funktionskatalogen zusammengeführt (vgl. Ronneberger 1974; Burkart 2002) und leiten sich aus einem übergeordneten „Dienst“ für die Gesellschaft ab (vgl. Rager / Weber 1992: 357). In Journalisten-Enqueten werden diese Funktionen als Leitbilder journalistischer Tätigkeiten operationalisiert und abgefragt, um daraus Schlüsse über die journalistische Orientierung der einzelnen Individuen oder in aggregierter Form über die Ausrichtung der Journalisten in einem Mediensystem zu ziehen (vgl. Scholl / Weischenberg 1998; Weischenberg et al. 2006; Donsbach / Patterson 2003; Weaver et al. 2007). In der Kommunikatorforschung gründet diese Vorgehensweise auf den Entstehungsbedingungen von Medieninhalten als der Leistung von Massenmedien (vgl. Esser 1998). Neben den individuellen Werten und Vorstellungen der Journalisten sind auch der gesellschaftliche und institutionelle Kontext entscheidende Determinanten. Auch die konzeptionell an das SCP Paradigma angelehnten Ansätze und Studien zur Medienperformanz in der Medienökonomie und politik argumentieren in ähnlicher Weise; Kontext, Marktstruktur und Marktverhalten bedingen die Leistung als Ergebnis eines Medienmarktes oder -systems (vgl. McQuail 1992; Wirth / Bloch 1995). Auch in diesen Forschungsrichtungen muss die Bewertungsgrundlage dabei nicht per se eine rein Ökonomische sein. Normen wie Vielfalt und Qualität, abgeleitet aus den gesellschaftlichen Funktionen, werden mindestens gleichwertig behandelt. Sowohl für die Kommunikatorforschung als auch für die Medienökonomie gilt daher, dass der Prozess hinter der Entstehung des Angebots, welcher die Leistung eines Mediensystems und damit dessen 
gesellschaftliche Wertschöpfung determiniert, von entscheidender Bedeutung ist.

Aus ökonomischer Sicht muss jedoch festgehalten werden, dass vom Medienmanagement in erster Linie nicht erwartet wird, dass gesellschaftliche Ansprüche erfüllt werden. Vielmehr gilt die Orientierung am ökonomischen Prinzip (vgl. Schumann / Hess 2006: 3) sowie am Markterfolg (vgl. Siegert 2002: 173). Die Bedienung der Nutzerpräferenzen ist dabei zentral. Es stellt sich daher für die Leistungserstellung und -erbringung die Frage nach den Zielen einer Organisation. Im Medienmanagement werden ökonomische und publizistische Formal- und Sachziele der Medienorganisationen analog dem dualen Gutscharakter unterschieden (vgl. Karmasin 1998; Chan-Olmsted 2006). Die Sachziele beschreiben den Gegenstandsbereich des Handelns, also die Herstellung, das Bündeln und Distribuieren von Medieninhalten (ökonomisch) von hoher publizistischer Qualität (publizistisch). Die Formalziele meinen den Zweck des Handelns (zugespitzt: Informieren vs. Gewinnmaximierung) und lehnen sich daher stark an die Funktionen an (vgl. Schumann / Hess 2006: 17; Sjurts 2005: 7). „In Medienunternehmen können Formalziele insbesondere betriebswirtschaftlichen oder publizistischen bzw. künstlerischen Ursprungs sein" (Schumann / Hess 2006: 17). Wie angedeutet, ist aus betriebswirtschaftlicher Sicht die Profitmaximierung jedoch übergeordnetes Ziel. Das Erwirtschaften von Einnahmen zur Deckung der anfallenden Kosten der Leistungserbringung, einschließlich einer Rendite für das eingesetzte Kapital, ist die Grundlage jeder privatwirtschaftlichen Organisation. Das entsprechende Ziel ist also fundamental. Ökonomische und publizistische Ziele müssen sich jedoch nicht diametral entgegenstehen, sie richten sich vereinfacht ausgedrückt an zwei organisatorisch getrennte Bereiche, die jeweils spezifische Leistungen erbringen (vgl. Altmeppen 2006). Entman (2005: 58) fasst die Ziele traditioneller Medienorganisationen entsprechend wie folgt zusammen: „(1) Reporting on important events, people, and issues, particularly those involving governmental institutions and actors, in ways that are accurate and balanced, and (2) generating sufficient advertising and circulation revenue to make a profit regarded by the stock market or private owners as acceptable". Auf Organisationsebene determinieren also die Zielsetzung und die Maßnahmen zur Zielverwirklichung die Wertschöpfung und Funktionserfüllung eines Mediensystems.

Dieses Spannungsfeld ist auch nicht nur in Modellen der journalistischen Einflusssphären oder in managementtheoretischen und industrieökonomischen Ansätzen zur Organisation und Leistung in einem Markt inte- 
griert. Vielmehr werden die Doppelfunktion von Massenmedien und eine unterstellte Zielhierarchie im Sinne ökonomischer vor publizistischer Ausrichtung auch in kritischen Debatten zur Medienleistung in demokratischen Gesellschaften (Medienkonzentration und Kommerzialisierung) diskutiert (vgl. Meier / Trappel 1998; Knoche 2001). Für den vorliegenden Beitrag ist jedoch weniger die paradigmatische Richtung zentral, sondern die Tatsache, dass sich die Leistungen von Medienorganisationen angebotsseitig auf organisationale Strukturen und strategische Ausrichtungen sowie Ziele zurückführen lassen.

Die ausschließliche Beschreibung von Leistungen und Ansprüchen oder deren Determinanten erlaubt allerdings noch keine Bewertung. Hierzu bedarf es einer Bewertungsgrundlage, welche aus den Funktionen normativ abgeleitet werden kann. Solche normativen Setzungen bedürfen zuerst der Klärung des funktionalen Bezugsrahmens (vgl. Burkart 2002: 381). Die Definition von Normen wie Qualität und Vielfalt werden aus dem Funktionskontext demokratisch verfasster Gesellschaften abgeleitet. Aus ökonomischer Sicht ist eine kollektive Setzung solcher Aufgaben, die ein System erfüllen soll, unter Verweis auf den methodologischen Individualismus zurückzuweisen (vgl. Heinrich 2010; Fu 2003). Die Konsumentenpräferenzen bilden die alleinige Bewertungsgrundlage. Anders als aus kritischer Warte heraus betrachtet (vgl. Jarren / Meier 2001), schließt die Nachfrageorientierung die Ausrichtung an gesellschaftlich relevanten Normen und Werten jedoch keineswegs aus. Gerade bei journalistisch ausgerichteten Medienorganisationen findet sich oft eine Wertorientierung, die sich stark mit journalistischen Werten und Normen deckt (vgl. Sylvie 2007; Weaver et al. 2007; Weischenberg et al. 2006; Patterson 1998). Im vorliegenden Beitrag wird daher ebenfalls die Nachfrage zur Beurteilung der gesellschaftlichen Wertschöpfung durch die Massenmedien herangezogen, weil erstens die Nachfrageorientierung der Medienorganisationen deren ökonomisches Bestehen gewährleistet und zweitens, weil auch gesellschaftliche Wertschöpfung und Aufgaben ihren Zweck für den demokratischen Prozess nur erfüllen, sofern die Leistung der Medien auf entsprechende Resonanz beim Publikum stößt (vgl. Frank / Klingler 1987).

\section{Forschungsfragen}

Wie dargelegt determinieren die Zielsetzungen von Medienorganisationen deren Leistungserbringung und damit die Wertschöpfung und Funktionserfüllung eines Mediensystems insgesamt. Entsprechend wird zunächst für die Angebotsseite folgende Forschungsfrage formuliert: 
Welche Ziele verfolgen Medienorganisationen und inwiefern berücksichtigen sie dabei Funktionen im Sinne gesellschaftlicher Wertschöpfung?

Um die Leistungs- und Funktionserfüllung umfassend abbilden zu können, muss in einem weiteren Schritt die Nachfrage einbezogen werden. Die Bedienung der Nutzerpräferenzen ist einerseits für Medienorganisationen zentral, andererseits aber auch für die Legitimation ihrer Leistung für die Gesellschaft. Somit zielt folgende Forschungsfrage auf die Nachfrageseite ab:

Wie beurteilt die Bevölkerung die Leistung und die Funktionserfüllung im Sinne gesellschaftlicher Wertschöpfung von Medienorganisationen?

\section{$4 \quad$ Methode und Operationalisierung}

Die in diesem Beitrag präsentierten Ergebnisse sind Teil eines interdisziplinären Forschungsprojektes ${ }^{1}$. Als Untersuchungsobjekte dienen die reichweitenstärksten, auf Informationsvermittlung fokussierten Tages-, Wochenzeitungen und TV-Programme sowie das Publikum in sechs europäischen Ländern. Die Länderauswahl folgt der Mediensystemtypologie von Hallin und Mancini (2004), und umfasst einen Vertreter des liberalen Typs (Großbritannien), drei demokratisch-korporatistische (Dänemark, Deutschland und Schweiz) sowie zwei polarisiert-pluralistische Mediensysteme (Italien und Frankreich). Diese Auswahl zielt auf eine Varianz der verschiedenen Kontexte ab und erlaubt es gegebenenfalls, Unterschiede in den Daten zu identifizieren und darauf zurückzuführen. Für die Auswahl regionaler Zeitungen und TV-Programme wurde das geographische Gebiet anhand der größten Metropolregionen in den untersuchten Ländern definiert: Berlin, Kopenhagen, London, Paris, Rom und Zürich. Metropolregionen wurden aufgrund ihrer wirtschaftlichen, politischen und kulturellen Relevanz als Ansatzpunkte für die regionale Eindämmung und Vergleichbarkeit der Angebote ausgewählt. Die Aus-

\footnotetext{
${ }^{1}$ Die Durchführung erfolgte innerhalb des nationalen Forschungsschwerpunkts NCCR Democracy „Herausforderungen für die Demokratie im 21. Jahrhundert“, gefördert durch den Schweizerischen Nationalfonds. Weitere Informationen finden sich unter: www.nccrdemocracy.uzh.ch.
} 
wahl der Medientitel folgte entsprechend der erwähnten Systematik: Reichweite und Fokus auf Informationsvermittlung in den jeweiligen Metropolregionen. In die Auswahl gehen daher regionale und nationale Angebote aus den Bereichen Tageszeitungen (Qualitäts-, Boulevard und Gratiszeitungen), Nachrichtenmagazine (wöchentlich) sowie Fernsehnachrichtensendungen öffentlicher oder privater Veranstalter ein. Insgesamt umfasst die Auswahl 100 Medientitel (ca. 12-19 pro Land).

Um die Ziele und Funktionen auf der Angebotsseite zu erfassen, wurden standardisierte Online-Befragungen mit Führungskräften (oder deren Stellvertreter) und Chefredakteuren (oder deren Stellvertreter) in allen sechs Metropolregionen durchgeführt. Die Termine für die Befragungen wurden zuvor vereinbart und das Ausfüllen des Fragebogens in der jeweiligen Landesprache (in Dänemark englisch) telefonisch begleitet. Beide Maßnahmen dienten dazu, das Engagement und den Rücklauf zu erhöhen. Insgesamt wurde ein zufriedenstellender Rücklauf von $58 \%$ $(\mathrm{N}=58)$ erzielt. Für die Schweiz $(\mathrm{N}=21$ inklusive deutsch und französisch sprachiger Titel), Dänemark $(\mathrm{N}=12)$ und Frankreich $(\mathrm{N}=11)$ trifft dies im Einzelnen zu, während in Deutschland $(\mathrm{N}=6)$ und Italien $(\mathrm{N}=6)$ weniger Interviews geführt werden konnten. In Großbritannien fiel der Rücklauf sehr gering aus $(\mathrm{N}=2)$. Dies lässt sich teilweise mit der Auswahl der Medienverantwortlichen erklären, da nur Führungskräfte und Chefredakteure in die Stichprobe eingeschlossen wurden und Zielpersonen vergleichbare Rollen in ihren Organisationen innehaben mussten.

Gleichzeitig zur Befragung der Medienverantwortlichen wurden in den Metropolregionen und auf nationaler Ebene repräsentative Publikumsbefragungen durchgeführt. Die Stichprobenauswahl und die Durchführung in der jeweiligen Landessprache wurden von einem internationalen Umfrageinstitut vorgenommen. In Bezug auf Alter, Geschlecht und Wohnort sind die Stichproben repräsentativ und setzen sich in den Metropolregionen und entsprechenden Ländern wie folgt zusammen: Berlin (regionale Stichprobe $\mathrm{N}=741$, nationale Stichprobe $\mathrm{N}=1482$ ), Kopenhagen $(\mathrm{N}=749, \mathrm{~N}=1489)$, London ( $\mathrm{N}=740, \mathrm{~N}=1494)$, Paris $(\mathrm{N}=741, \mathrm{~N}=1495)$, Rom ( $N=738, N=1459)$, und Zürich $(\mathrm{N}=748, \mathrm{~N}=1523)$. Die Befragung erfolgte innerhalb von vier Monaten in zwei Wellen (Panel). In der ersten Welle wurde der Fokus auf die Einschätzungen bezüglich Printtiteln gelegt, während in der zweiten Welle die Einschätzung der meistgenutzten TV-Programme abgefragt wurde.

Publikum und Medienverantwortlichen wurden dieselben Items vorgelegt. Die Organisationsziele (vgl. Weaver et al. 2007; Demers 1996) bil- 
den hier natürlich eine Ausnahme. Die Einschätzung des Publikums und der Medienverantwortlichen betreffend die gesellschaftliche Wertschöpfung und Funktionserfüllung wurden mittels sogenannter „, functional value "Items abgefragt, die bereits in repräsentativen Journalistenumfragen angewandt wurden (vgl. Weaver et al. 2007; Weischenberg et al. 2006). Die Medienverantwortlichen wurden gebeten, ihre eigenen Medientitel zu bewerten: „Bitte geben Sie an, inwiefern die folgenden gesellschaftlichen Aufgaben von Ihrem Medium erfüllt werden“. Diese Frage wurde nur Chefredaktoren gestellt oder für den Fall, dass kein Chefredakteur für das Interview zur Verfügung stand, der wirtschaftlich verantwortlichen Führungskraft $(\mathrm{N}=38)$. Dem Publikum wurde dieselbe Frage bezüglich ihrer bevorzugten Zeitungstitel und Fernsehnachrichtenprogramme vorgelegt. Für die Beantwortung der Fragen wurde jeweils eine fünfstufige Likert Skala „1 - unwichtig“ bis , 5 - sehr wichtig“ herangezogen. Um Recency- und Primacy-Effekten entgegenzuwirken, wurden die Items zufällig rotiert. $\mathrm{Da}$ es sich bei den Antworten der Medienverantwortlichen um Selbsteinschätzungen handelt, ist dem Problem sozialer Erwünschtheit der Antwortvergabe im Sinne einer Überbewertung gesellschaftlich relevanter Aufgaben und Ziele Rechnung zu tragen. Es ist zudem angebracht von Selbsteinschätzung zu sprechen, denn es handelt sich nicht um eine Betrachtung real erbrachter Leistungen. Für den Vergleich der Angebots- und Nachfrageseite werden ANOVA Mittelwertvergleiche durchgeführt.

\section{$5 \quad$ Ergebnisse}

Zunächst werden die Gewichtungen der unterschiedlichen Organisationsziele betrachtet, weil sie als Basis der Leistungserbringung und somit der Funktionserfüllung fungieren. Anschließend werden die Selbst- und Fremdwahrnehmung bezüglich der Funktionen im Sinne gesellschaftlicher Wertschöpfung der untersuchten Medienunternehmen analysiert. Die Einschätzungen der Medienverantwortlichen zur Wichtigkeit unterschiedlicher Zielsetzungen (vgl. Tabelle 1) zeigen, dass Gewinnziele leicht hinter publizistischen Zielen stehen. So zeigt die Betrachtung der Mittelwerte über alle Kontexte und Mediengattungen hinweg, dass „,journalistische Arbeit in hoher, überdurchschnittlicher Qualität", mit einem Mittelwert von 4,60 als am wichtigsten bewertet wurde. Demgegenüber rangiert das Ziel „hoher, überdurchschnittlicher Gewinn“ $(3,60)$ an letzter Stelle. Auch die Beeinflussung der politischen Tagesordnung und Themensetzung $(3,66)$ wird als wenig relevantes Ziel angesehen. Das ebenfalls ökonomisch relevante Ziel „Ansprache eines möglichst großen Publikums“ $(4,31)$ wurde hingegen als zweitwichtigstes benannt. Betrach- 
tet man diese Ergebnisse unter Einbezug der jeweils erhobenen Standardabweichung, ist eine Differenzierung der ermittelten Werte nötig. Während sich die Medienverantwortlichen gesamthaft bezüglich der Wichtigkeit der journalistischen Arbeit weitgehend einig sind, deuten die Standardabweichungen betreffend Meinungsführerschaft $(1,3)$ und hoher, überdurchschnittlicher Gewinn $(1,4)$ auf heterogene Einschätzungen hin. Eine differenzierte Betrachtung der Ergebnisse erlaubt die Auswertung nach Mediengattung: Während die Bewertungen bezüglich der Meinungsführerschaft durch die Medienverantwortlichen für Print $(\mathrm{MW}=$ 3,64) und TV (MW = 3,69) homogen ausfallen, weißt die Standardabweichung $(1,2$ und 1,6) dennoch auf gewisse Unterschiede der einzelnen Bewertungen hin. Eine mögliche Erklärung hierfür findet sich in der $\mathrm{Zu}-$ sammensetzung der Stichprobe.

So ist im Bereich TV eine deutliche Dominanz von Medienorganisationen mit einem öffentlichen Auftrag zu verzeichnen. Gesamthaft sind elf Medienverantwortliche einer solchen zuzuordnen, während nur zwei Experten einer rein privat organisierten Medienorganisation entstammen. Dadurch lässt sich unter Einbezug der Besonderheiten des öffentlichen Fernsehens partiell die Heterogenität der Einschätzung erklären. So kann vor allem im Bereich der Nachrichtensendungen die Informationsvermittlung und neutrale Berichterstattung als ein zentrales Element des öffentlichen Auftrages gesehen werden. Das Erstreben einer politischen Meinungsführerschaft scheint vor diesem Hintergrund von geringerer Relevanz vor allem für die öffentlichen Fernsehsender. Stärkere Abweichungen lassen sich betreffend der Einschätzungen des hohen, überdurchschnittlichen Gewinns aufzeigen. So wird nicht nur die Wichtigkeit der Gewinnziele für die Mediengattung Print $(M W=3,98)$ im Vergleich zu TV (MW = 2,31) deutlich höher eingeschätzt, Erstere weißt auch eine deutlich geringere Standardabweichung auf $(0,9)$ als innerhalb des Mediums TV $(2,0)$. Dies wird durch die Organisations- und Finanzstruktur von öffentlichen Sendern bedingt, die nicht auf Gewinn hin ausgerichtet sind. Privat organisierte Medienunternehmen können dagegen nicht auf eine Gebührenfinanzierung zurückgreifen, sondern müssen sich hauptsächlich über Werbeeinnahmen finanzieren, weshalb sie Gewinnziele höher bewerten.

Dass die Funktionserfüllung im Sinne gesellschaftlicher Wertschöpfung für alle Fernsehsender eine wichtige Rolle spielt, zeigen die Bewertungen zum Ziel ,journalistische Arbeit in hoher, überdurchschnittlicher Qualität“". Diesem Ziel wurde durchweg von allen Befragten, auch von denen des privaten Sektors, die höchste Wichtigkeit attestiert $(5,00)$. Dies zeigt, 
dass nicht nur die öffentlichen, sondern auch die privaten Sender, bezüglich ihrer Nachrichtensendungen ein primäres Qualitätsziel verfolgen. Auch die Einzelauswertung im Bereich Print zeigt, dass Qualitätsziele eindeutig am höchsten bewertet wurden $(4,49)$, diese jedoch in den Einschätzungen hinter dem Bereich TV etwas zurückstehen und auch weniger homogen ausgefallen sind $(0,8)$. Insgesamt lässt sich somit festhalten, dass die Medienverantwortlichen mit ihren Einschätzungen betreffend der Zielsetzung ein deutliches Zeichen für die Qualität der journalistischen Arbeit setzen.

Tabelle 1: Relevanz der Ziele der untersuchten Medienunternehmen

\begin{tabular}{lccc}
\hline Ziele & $\begin{array}{c}\text { Mittelwert } \\
(\mathrm{SA}) \\
\text { Gesamt } \\
(\mathrm{N}=58)\end{array}$ & $\begin{array}{c}\text { Mittelwert } \\
(\mathrm{SA}) \\
\text { Print } \\
(\mathrm{N}=45)\end{array}$ & $\begin{array}{c}\text { Mittelwert } \\
\text { (SA) } \\
\text { TV } \\
(\mathrm{N}=13)\end{array}$ \\
$\begin{array}{l}\text { Hoher, überdurchschnittlicher } \\
\text { Gewinn }\end{array}$ & $3,60(1,4)$ & $3,98(0,9)$ & $2,31(2,0)$ \\
$\begin{array}{l}\text { Hohe Arbeitsmoral der Mitarbei- } \\
\text { ter sichern }\end{array}$ & $4,26(0,7)$ & $4,18(0,6)$ & $4,54(0,7)$ \\
$\begin{array}{l}\text { Ansprache möglichst großen Pub- } \\
\text { likums }\end{array}$ & $4,31(0,9)$ & $4,24(1,0)$ & $4,54(0,8)$ \\
$\begin{array}{l}\text { Politische Tagesordnung beein- } \\
\text { flussen und Themen setzen }\end{array}$ & $3,66(1,3)$ & $3,64(1,2)$ & $3,69(1,6)$ \\
$\begin{array}{l}\text { Journalistische Arbeit in hoher } \\
\text { Qualität }\end{array}$ & $4,60(0,7)$ & $4,49(0,8)$ & $5,00(0,0)$ \\
\hline
\end{tabular}

Quelle: Eigene Darstellung

Im Folgenden werden die Angebotsseite und die Nachfrageseite zusammengeführt. Aufgrund der unterschiedlichen Gruppengrößen der Medienverantwortlichen und des Publikums ist bei der Interpretation der Ergebnisse der ANOVA oder t-Tests Vorsicht geboten. Dieser Umstand erlaubt auch keine Feinsegmentierung innerhalb der einzelnen Länder geschweige denn nach Mediengattungen. Eine Aggregatsbetrachtung kann allerdings durchaus vorgenommen werden. Die Einführung einer Kovariaten in die ANOVA Mittelwertvergleiche kann zudem einen wenn auch beschränkten Rückschluss auf mögliche Ländereffekte geben. 
Abbildung 1: Aggregierte Bewertungen gesellschaftlicher Aufgaben von Medienverantwortlichen und Publikum

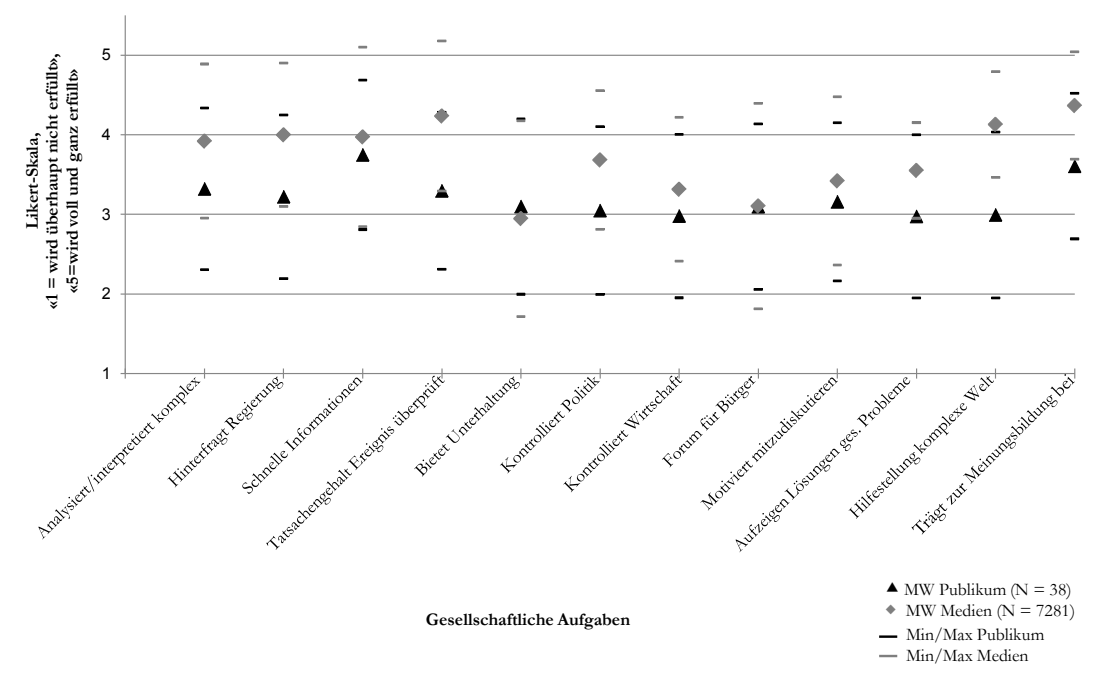

Quelle: Eigene Darstellung

Abbildung 1 zeigt einen Überblick über die Bewertungen der gesellschaftlichen Aufgaben. Vergleicht man auf dieser Basis die Einschätzungen der Medienverantwortlichen mit jenen des Publikums über alle Länder hinweg, fällt auf, dass Erstere ihre Leistungen durchwegs besser beurteilen. Einzig das Item „bietet Unterhaltung“ bewerten die Rezipienten besser als die Medienverantwortlichen. Dieses Item wird von den Führungskräften als am wenigsten relevant erachtet. Insgesamt sehen alle Führungskräfte ihre gesellschaftlichen Aufgaben vor allem in der Erbringung eines Beitrags zur gesellschaftlichen Meinungsbildung (MW = 4,37), des Berichtens über Ereignisse, deren Tatsachengehalt überprüft werden kann $(4,24)$, sowie der Hilfe für die Rezipienten, sich in einer komplexen Welt zurechtzufinden (4,13). Die dargestellten Intervalle (MW \pm Standardabweichung ${ }^{2}$ ) zeigen allerdings auch, dass die Einschätzungen durchaus variieren. Auch die Antworten der Rezipienten variie-

\footnotetext{
${ }^{2}$ Bei angenommener Normalverteilung entspricht dieses Intervall knapp 70 Prozent der Antwortvergaben $(68,3 \%)$.
} 
ren mit einer Standardabweichung von durchschnittlich ca. 1. Insgesamt zeigen sich hier allerdings weniger große Unterschiede in der Gewichtung der einzelnen Items ${ }^{3}$ und gemessen an den Skalenwerten sind die Rezipienten mit den Leistungen der Medien recht zufrieden.

Die angesprochenen Unterschiede zwischen der Leistungsbewertung des Publikums und der Medienverantwortlichen sind für einzelne gesellschaftliche Aufgaben deutlicher als für andere. Für die Aufgaben „vermittelt möglichst schnell Information“, „bietet Unterhaltung und Entspannung“", ,kontrolliert die Verantwortlichen in der Wirtschaft und ihre Handlungen“, „motiviert normale Leute, bei wichtigen Themen öffentlich mitzudiskutieren“ sowie „gibt normalen Leuten eine Chance, ihre Meinung über Themen von öffentlichem Interesse zum Ausdruck zu bringen" sind nur geringe Unterschiede in den Bewertungen beider Gruppen auszumachen. Es handelt sich dabei um die Aufgaben, welche von allen Chefredakteuren am niedrigsten bewertet wurden. Diese augenscheinlich geringen oder kaum vorhandenen Unterschiede werden durch statistische Mittelwertvergleiche bestätigt (Tabelle 2). Einzig die Aufgabe „kontrolliert Verantwortliche der Wirtschaft“ bewerten die Chefredaktoren leicht besser als die Rezipienten. Für die übrigen vier angedeuteten Aufgaben ergeben sich keine signifikanten Gruppenunterschiede.

${ }^{3}$ Dies ist mitunter auch auf die markant grössere Fallzahl zurückzuführen. 
Tabelle 2: Vergleich der Funktionen im Sinne gesellschaftlicher Wertschöpfung aus Sicht von Publikum und Medienverantwortlichen

\begin{tabular}{|c|c|c|c|c|}
\hline Gesellschaftliche Aufgaben & $\begin{array}{l}\text { ANOVA } \\
\text { (F-Wert) }\end{array}$ & $\begin{array}{c}\text { t-Test } \\
\text { unabh. SP } \\
\text { (T-Wert) }\end{array}$ & $\begin{array}{c}\text { Faktor } \\
\text { «Medien / } \\
\text { Publikum» }\end{array}$ & $\begin{array}{c}\text { Kovariate } \\
\text { «Mediensystem» }\end{array}$ \\
\hline Analysiert/interpretiert komplexe Sachverh. & $15,22^{* * *}$ & & $13,00 * * *$ & $20,79 * * *$ \\
\hline Hinterfragt Regierung & $21,64 * * *$ & & $21,29 * * *$ & $52,86^{* * *}$ \\
\hline Schnelle Informationen & n.s. & & & \\
\hline Tatsachengehalt Ereignis überprüft & $34,33 * * *$ & & $34,70^{* * *}$ & $12,92 * * *$ \\
\hline Bietet Unterhaltung & n.s. & & & \\
\hline Kontrolliert Politik & $13,78^{* * *}$ & & $13,51 * * *$ & $37,65^{* * *}$ \\
\hline Kontrolliert Wirtschaft & $4,05^{*}$ & & $3,91 *$ & $23,87 * * *$ \\
\hline Forum für Bürger & & n.s. & & - \\
\hline Motiviert mitzudiskutieren & n.s. & & & \\
\hline Aufzeigen Lösungen ges. Probleme & $12,02 * *$ & & $11,84 * * *$ & $16,11 * * *$ \\
\hline Hilfestellung in komplexer Welt & & $-10,49 * * *$ & & - \\
\hline Trägt zur Meinungsbildung bei & & $-6,93 * * *$ & & - \\
\hline
\end{tabular}

Quelle: Eigene Darstellung; ${ }^{*} \mathrm{p}<0,1,{ }^{* *} \mathrm{p}<0,05,{ }^{* * *} \mathrm{p}<0,01$ 
Die signifikantesten Unterschiede finden sich für die Items „,berichtet nur über Ereignisse, deren Tatsachengehalt überprüft werden kann", „hinterfragt Behauptungen und Aussagen der Regierung“, „analysiert und interpretiert komplexe Sachverhalte“, sowie „kontrolliert die Verantwortlichen in der Politik und deren Handlungen“. Ebenfalls hochsignifikante Unterschiede ergeben die t-Tests zu den Items „hilft den Lesern/Zuschauern, sich in einer komplexen Lebenswelt zurechtzufinden" und „trägt zur gesellschaftlichen Meinungsbildung bei“. T-Tests für unabhängige Stichproben wurden durchgeführt, weil die Voraussetzung der Varianzhomogenität bei den entsprechenden Items nicht gegeben ist. Insgesamt zeigt sich, dass für die meisten gesellschaftlichen Aufgaben signifikante Unterschiede zwischen der Selbsteinschätzung der Medienverantwortlichen und der Fremdeinschätzung durch das Publikum vorliegen.

Eine Länderauswertung kann aufgrund der kleinen Fallzahl auf der Angebotsseite nicht durchgeführt werden. In Anlehnung an die der Länderauswahl zugrunde liegende Typologie kann allerdings die Einführung der Kovariaten „Mediensystem“ darüber Auskunft geben, ob die Zugehörigkeit zu einem demokratisch-korporatistischen System Einfluss auf die Bewertung der gesellschaftlichen Aufgaben hat und ob nach der Kontrolle der Mediensystemzugehörigkeit die Gruppenunterschiede zwischen Medienverantwortlichen und Publikum im Aggregat bestehen bleiben. Die Kovariate ist eine Dummy-Variable mit den Ausprägungen $1=$ demokratisch-korporatistisches Mediensystem und $0=$ polarisiertpluralistisches Modell. Die Auspartialisierung möglicher Mediensystemeffekte (letzte Spalte in Tabelle 2) ändert nichts an den zuvor rapportierten Gruppenunterschieden. Allesamt bleiben sie vorhanden (zweitletzte Spalte in Tabelle 2). Anhand des Einbezugs der Kovariaten Mediensystem ist jedoch noch nicht geklärt, wie sich die Länder und Gruppen im Einzelnen unterscheiden. Die entsprechenden Ergebnisse können nur wie folgt interpretiert werden: Die Bewertungen der gesellschaftlichen Aufgaben erfolgen in den beiden Typen von Mediensystemen unterschiedlich und die Unterschiede zwischen den Selbsteinschätzungen durch die Medienverantwortlichen und den Fremdeinschätzungen durch das Publikum können weiterhin festgestellt werden.

Ein Blick in die Daten vermag dennoch aufzuzeigen, dass sowohl zwischen den Ländern Unterschiede bestehen, als auch innerhalb der untersuchten Länder Gruppenunterschiede erkennbar sind. Die höhere Einschätzung der Medienverantwortlichen ist über alle Länder und Aufgaben hinweg zu konstatieren. Einzig die Aufgabe „vermittelt Informatio- 
nen möglichst schnell “ wird in Dänemark und Italien auch von den Chefredakteuren besser bewertet als vom Publikum. Gleiches gilt in Italien für die Unterhaltungs- und Entspannungsfunktion der Medien. Die Chefredakteure gewichten diese Aufgabe leicht höher als die italienischen Rezipienten. Die dänischen Chefredakteure sind ebenfalls eine Ausnahme die Forumsfunktion betreffend, welche in allen anderen Ländern von Publikum und Medienverantwortlichen relativ tief bewertet wurde. Das dänische Publikum ist im Ländervergleich mit der Kontrollfunktion der Politik und Wirtschaft gegenüber zufriedener und bewertet diese Aufgabe für die Wirtschaft sogar besser als dies die dänischen Medienverantwortlichen tun. Diese Erläuterungen sind rein qualitativer Natur und basieren auf augenscheinlichen Vergleichen zwischen den Gruppen und den untersuchten Ländern mittels deskriptiver Statistik. Die Aussagen sind daher als Tendenzen aufzufassen.

\section{Diskussion}

Anhand der präsentierten Ergebnisse lässt sich zur Beantwortung der ersten Forschungsfrage festhalten, dass Medienorganisationen, neben betriebswirtschaftlichen Zielsetzungen, journalistische Werte und Funktionen im Sinne gesellschaftlicher Wertschöpfung fest in ihrem Selbstverständnis verankert haben. Letztere sind zumindest gleichwertig. Besonders deutlich hebt sich der hohe Qualitätsanspruch bezüglich journalistischer Arbeit hervor, sowohl im Printbereich, als auch über die öffentlichen und privaten Fernsehsender hinweg. Der vermeintliche absolute Vorrang betriebswirtschaftlicher Zielsetzung wird in der vorliegenden Untersuchung damit nicht gestützt.

Selbst wenn eine gewisse soziale Erwünschtheit im Antwortverhalten der befragten Führungskräfte und Chefredakteure angenommen werden darf, erstaunt dieses Ergebnis dennoch. Ansatzweise lässt es sich durch die Berufsrollen und beruflichen Orientierungen von Journalisten erklären (vgl. Marr et al. 2001; Weischenberg et al. 2006; Kaltenbrunner et al. 2007). Wie z.B. Marr et al. (2001: 123) zeigen, verstehen sich Journalisten vor allem als neutrale Berichterstatter, Analytiker und Kritiker und entsprechen damit eher der Tatsachen- bzw. Gemeinwohlorientierung. Diese Berufsrollenorientierung könnte auch bei den befragten Führungskräften und Chefredakteuren durchschlagen.

Bezüglich der Leistung und Funktionserfüllung im Sinne gesellschaftlicher Wertschöpfung von Medienorganisationen (Forschungsfrage 2) zeichnet sich stellenweise eine Diskrepanz zwischen der Selbstwahrneh- 
mung der Medienverantwortlichen und dem Publikum ab. Zwar scheinen sich Erstere ihrer gesellschaftspolitischen Aufgaben bewusst, doch wird ihre Aufgabenerfüllung vom Publikum nicht in gleichem Ausmaß wahrgenommen. Hierbei lassen sich sowohl länderspezifische Unterschiede, wie auch Differenzen innerhalb der einzelnen Länder zwischen den Gruppen festhalten. Dieses Ergebnis ist insbesondere im Zuge struktureller Veränderung und vor dem Hintergrund der Forderung der Rücksichtnahme auf traditionelle Massenmedien (z.B. in Form eines Leistungsschutzrechts) von Interesse. So sollte ein postulierter öffentlicher Mehrwert beim Publikum auch auf Resonanz stoßen.

Im Hinblick auf die Nachfrageorientierung sind die ermittelten Ergebnisse ebenfalls interessant. Gerade wenn man davon ausgeht, dass bei journalistisch ausgerichteten Medienorganisationen eine Nachfrageorientierung die Ausrichtung an gesellschaftlich relevanten Normen und Werten nicht ausschließt, deuten die Ergebnisse unter ökonomischen Aspekten eine gewisse Schieflage an. Zwar ist das Publikum mit der Leistungserbringung der Medien durchaus zufrieden, bewertet diese jedoch (erwartungsgemäß) schlechter als die Medienverantwortlichen selbst. Dabei kann einerseits von einer gewissen sozialen Erwünschtheit im Antwortverhalten der befragten Führungskräfte und Chefredakteure und andererseits von einer weder durch die Vertriebspreise noch durch die Niedrigkostensituation der Mediennutzung gerechtfertigten Anspruchshaltung des Publikums ausgegangen werden. Dennoch besteht auf Seiten der Medienorganisationen, auch unter ökonomischen Aspekten, ein Nachbesserungsbedarf bezüglich der Erfüllung relevanter Funktionen im Sinne gesellschaftlicher Wertschöpfung, wenn diese als Bestandteil der Nachfrageorientierung verstanden werden.

\section{Literatur}

Altmeppen, Klaus-Dieter (2006): Journalismus und Medien als Organisationen. Leistungen, Strukturen und Management. Verlag für Sozialwissenschaften: Wiesbaden.

Bonfadelli, Heinz (2010): Was ist öffentliche Kommunikation? S. 111-142 in: Bonfadelli, Heinz / Jarren, Otfried / Siegert, Gabriele (Hrsg., 2010): Einführung in die Publizistikwissenschaft. Haupt: Bern.

Burkart, Roland (2002): Kommunikationswissenschaft. Grundlagen und Problemfelder / Umrisse einer interdisziplinären Sozialwissenschaft. 2259. Band. Böhlau: Wien.

Chan-Olmsted, Sylvia M. (2006): Competitive strategy for media firms. Strategic and brand management in changing media markets. Lawrence Erlbaum Associates: Mahwah, NJ.

Demers, David P. (1996): Corporate newspaper structure, profits, and organizational goals. S. 1-23 in: Journal of Media Economics. 2, 9. Routledge: London. 
Donsbach, Wolfgang / Patterson, Thomas E. (2003): Journalisten in der politischen Kommunikation: Professionelle Orientierungen von Nachrichtenredakteuren im internationalen Vergleich. S. 281-304 in: Pfetsch, Barbara / Esser, Frank (Hrsg., 2003): Politische Kommunikation im internationalen Vergleich: Grundlagen, Anwendungen, Perspektiven. VS Verlag: Wiesbaden.

Entman, Robert M. (2005): The nature and sources of news. S. 48-65 in: Overbolser, Geneva (Hrsg., 2005): The press. Oxford University Press: Oxford, New York.

Esser, Frank (1998): Die Kräfte hinter den Schlagzeilen. Englischer und deutscher Journalimus im Vergleich. 23. Band. Alber: Freiburg (Breisgau).

Frank, Bernward / Klingler, Walter (1987): Die veränderte Fernsehlandschaft. Zwei Jahre ARD/ZDF-Begleitforschung zu den Kabelpilotprojekten. 7. Band. Frankfurt am Main.

Fu, W ayne (2003): Applying the structure-conduct-performance framework in the media industry analysis. S 275-284 in: International Journal on Media Management. 4, 5.

Hallin, Daniel C. / Mancini, Paolo (2004): Comparing media systems. Three models of media and politics. Cambridge University Press: Cambridge.

Heinrich, Jürgen (2010): Medienökonomie. Band 1: Mediensystem, Zeitung, Zeitschrift, Anzeigenblatt. VS Verlag: Wiesbaden.

Jarren, Otfried / Meier, Werner A. (2001): Ökonomisierung der Medienindustrie: Ursachen, Formen und Folgen. S. 145-158 in: Medien \& Kommunikationswissenschaft. 2, 49. Nomos: Baden-Baden.

Kaltenbrunner, Andy / Karmasin, Matthias / Kraus, Daniela / Zimmermann, Astrid / Leitner, Judith (2007): Der Journalisten-Report. Österreichs Medien und ihre Macher - Eine empirische Erhebung. Facultas: Wien.

Karmasin, Matthias (1998): Medienökonomie als Theorie (massen-)medialer Kommunikation. Kommunikationsökonomie und Stakeholder Theorie. Nausner \& Nausner: Graz.

Knoche, Manfred (2001): Kapitalisierung der Medienindustrie aus politökonomischer Perspektive. S. 177-194 in: Medien \& Kommunikationswissenschaft. 2, 49. Nomos: Baden-Baden.

Marr, Mirko / Wyss, Vinzenz / Blum, Roger / Bonfadelli, Heinz (2001): Journalisten in der Schweiz. Eigenschaften, Einstellungen, Einflüsse. 13. Band. UVK Verlagsgesellschaft: Konstanz.

McQuail, Denis (1992): Media performance. Mass communication and the public interest. Sage: London.

Meier, Werner A. / Trappel, Josef (1998): Media concentration and the public interest. S. 177-194 in: McQuail, Denis / Siune, Karen (Hrsg., 1998): Media policy. Convergence, concentration and commerce. Sage: London.

Patterson, Thomas E. (1998): Political roles of the journalist. S. 17-32 in: Graber, Doris A. / McQuail, Denis / Norris, Pippa / Graber, Doris (Hrsg., 1998): The politics of news. The news of politics. CQ Press: Washington DC.

Rager, Günther / Weber, Bernd (1992): Publizistische Vielfalt zwischen Markt und Politik. S. 357-366 in: Media Perspektiven. 6, 10. Frankfurt.

Ronneberger, Franz (1974): Die politischen Funktionen der Massenkommunikation, S. 193 205 in: Langenbucher, Wolfgang R. (Hrsg., 1974): Zur Theorie der politischen Kommunikation. Piper: München.

Scholl, Armin / Weischenberg, Siegfried (1998): Journalismus in der Gesellschaft. Theorie, Methodologie und Empirie. Westdeutscher Verlag: Opladen.

Scbumann, Matthias / Hess, Thomas (2006): Grundfragen der Medienwirtschaft. Eine betriebswirtschaftliche Einführung. Springer: Berlin. 
Siegert, Gabriele (2002): Medienmanagement als Marketingmanagement, S. 173-195 in: Karmasin, Matthias / Winter, Carsten (Hrsg., 2002): Grundlagen des Medienmanagements. UTB: München.

Sjurts, Insa (2005): Strategien in der Medienbranche. Grundlagen und Fallbeispiele. Gabler: Wiesbaden.

Sylvie, George (2007): Decision making by nordic newspaper editors. An exploratory study and comparison to US editors. JIBS: Jönköping.

Weaver, David H. / Beam, Randal A. / Brownlee, Bonnie J. / Voakes, Paul S. / Wilhoit, G. Cleveland (2007): The American journalist in the 21st century. US news people at the dawn of a new millennium. Lawrence Erlbaum Associates: Mahwah, NJ.

Weischenberg, Siegfried / Malik, Maja / Scholl, Armin (2006): Die Souffleure der Mediengesellschaft. Report über die Journalisten in Deutschland. UVK Verlagsgesellschaft: Konstanz.

Wirth, Michael O. / Bloch, Harry (1995): Industrial organization theory and media industry analysis. S. 15-26 in: Journal of Media Economics. 2, 8. Routledge: London. 